\title{
Design of Optimal Scheduler for Process Scheduling
}

\author{
Sambath M, D. John Aravindhar, P. Harshavardhan Reddy, Lokesh Kumar Ravipati, Suthapalli \\ Sai Gowtham, Korrapati Jagadeesh, Vemula Raviteja
}

\begin{abstract}
CPU Scheduling takes plays an important role in multiprogramming systems. There are several programs present in memory. It is the responsibility of operating systems to select the process and assign it to CPU. There are various algorithms available for CPU Scheduling. The algorithm's performance depends on various factors like arrival time, priority etc. This paper helps to select the best algorithm by comparing various algorithms under the same condition and analyzed them based on various factors like waiting time, turnaround time, CPU utilization, Throughput.
\end{abstract}

Keywords: scheduler, throughput, turnaround time

\section{INTRODUCTION}

In earlier days the processors are capable of executing a single program at a time. The CPU utilization is very less and also it took lot of time to complete the process. When there are multiple programs it has to execute one by one. In recent days multi programming system are capable of executing multiple programs at a time. It improves the CPU utilization as well as consumes less time to complete the process.

\section{CPU SCHEDULER}

The CPU scheduler or short term scheduler selects the process from the ready queue and assign it to the CPU for execution. The process will be moving from ready to running state (Figure 1). When the process waiting for any event and $\mathrm{I} / \mathrm{O}$ to happen, then the process is moved to waiting state. Upon completion of $\mathrm{I} / \mathrm{O}$ or event, the process is again coming back to ready state and moved to running state by the

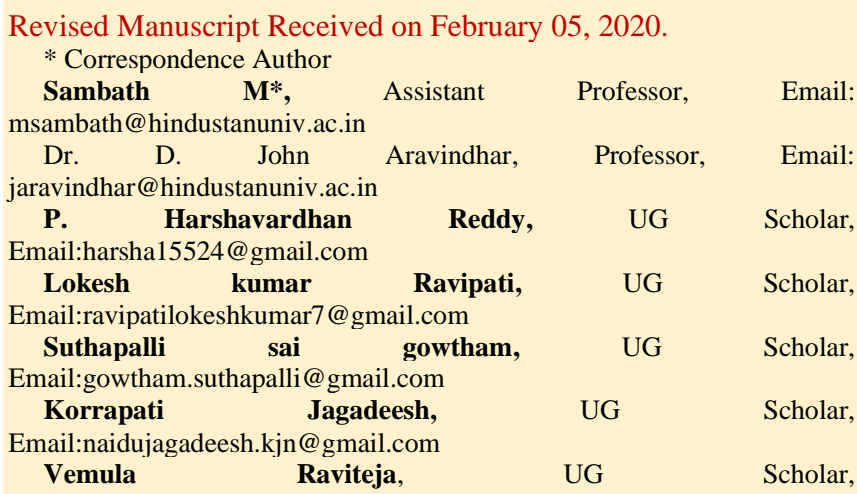
Email:chowdaryraviteja9999@gmail.com Department of Computer Science \& Engineering, Hindustan Institute of Technoogy and Science, Chennai, India.

(C) The Authors. Published by Blue Eyes Intelligence Engineering and Sciences Publication (BEIESP). This is an open access article under the CC BY-NC-ND license (http://creativecommons.org/licenses/by-nc-nd/4.0/) scheduler when the CPU is available. If the process gets completed then it is moved to the terminated state.[1]

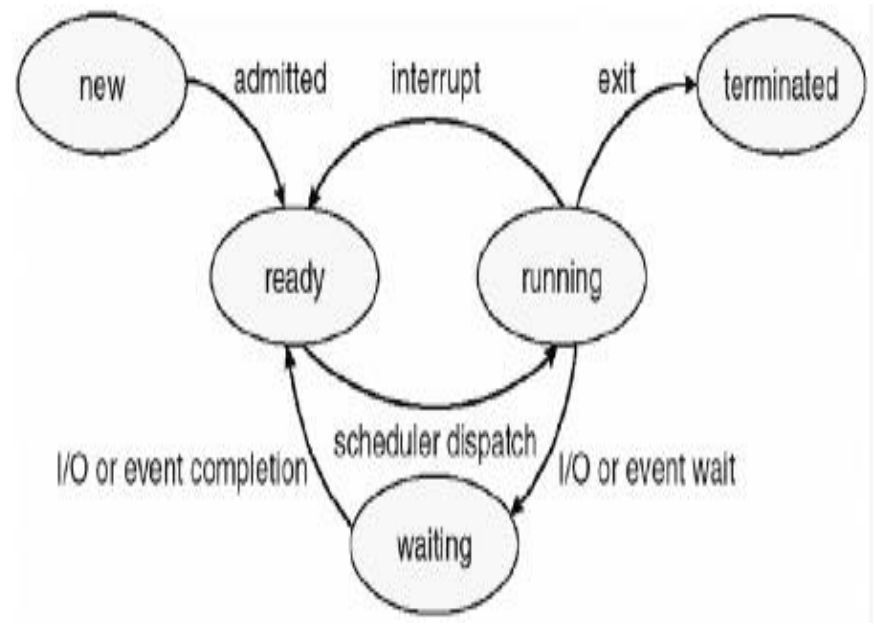

Figure 1 Process scheduling

The best CPU scheduler select the best algorithm in such a way that it leads to minimum turnaround time, minimum waiting time, minimum response time, maximum CPU utilization rate and maximum throughput. The Scheduling algorithms available are:[2]

1. First come first served(FCFS) scheduling

2. Shortest job first scheduling(SJF).

3. Priority scheduling.

4. Round Robin scheduling(RR).

The performance of the scheduling algorithm is estimated based on various criteria like CPU utilization, Throughput, Turnaround time, waiting time and response time.[3]

1. CPU utilization indicates how effectively CPU is utilized.

2. Throughput denotes the number of processes completed per unit time.[4]

3. Turnaround Time is the time needed for the process to complete its execution.[5]

4. Waiting Time is the amount of time a process waiting in the queue.

5. Response Time is the time between submission of request for execution and till the first response is produced not the output.

\section{FCFS SCHEDULING}

The processes are loaded from memory into ready queue. From the ready queue the processes are taken based on their arrival time and assigned to the processor for execution. 


\section{Design of Optimal Scheduler for Process Scheduling}

[6] The table 1 lists the processes and their execution time (burst time). The FCFS scheduling (Figure 2) is applied and the average waiting time and turnaround time are calculated and it is listed in table 1.

Table 1 Process and their burst times

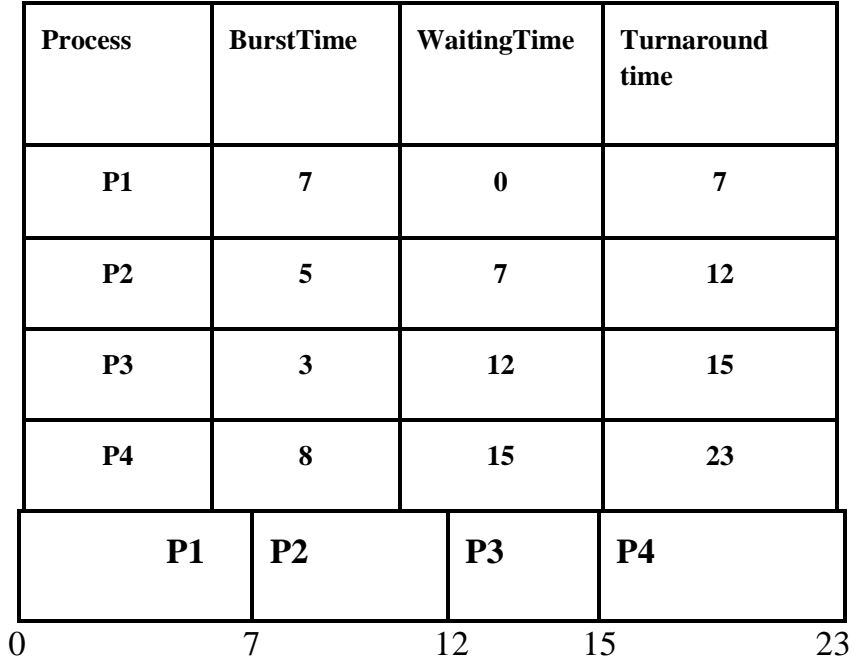

Figure 2 FCFS Scheduling

waiting time for $\mathrm{P} 1=0, \mathrm{P} 2=7, \mathrm{P} 3=12, \mathrm{P} 4=15$

Average waiting time $=(0+7+12+15) / 4=8.5$.

Average TurnaroundTime $=(7+12+15+23) / 4=14.25$.

\section{SJF SCHEDULING}

The processes are selected in the order of the burst time and assigned to CPU for execution. [8]

The table 2 lists the processes and their execution time (burst time). The SJF scheduling (Figure 3) is applied and the average waiting time and turnaround time are calculated and it is listed in table 2 .

Table 2 Process and their burst times

\begin{tabular}{|c|c|c|c|}
\hline Process & BurstTime & WaitingTime & $\begin{array}{l}\text { Turnaround } \\
\text { time }\end{array}$ \\
\hline P1 & 7 & 8 & 15 \\
\hline P2 & 5 & 3 & 8 \\
\hline P3 & 3 & $\mathbf{0}$ & 3 \\
\hline P4 & 8 & 15 & 23 \\
\hline P3 & P2 & P1 & P4 \\
\hline
\end{tabular}

Average waiting time $=(8+3+0+15) / 4=6.5$.

Average TurnaroundTime $=(15+8+3+23) / 4=12.25$.

\section{PRIORITY SCHEDULING}

In this type of scheduling additional input called priority is given for each process. The process which is having the highest priority will be selected and assigned to the CPU for execution. The starvation problem is the main issue in ths kind of scheduling. i.e., low priority processes will never execute. This problem can be solved the method of aging. Aging technique increases the priority of the processes as the time progresses in the queue.[7]

The table 3 lists the processes and their execution time (burst time). The priority scheduling (Figure 4) is applied and the average waiting time and turnaround time are calculated and it is listed in table 3.

Table 3 Process, burst times and priority

\begin{tabular}{|c|c|c|c|c|}
\hline Process & $\begin{array}{l}\text { Burst } \\
\text { Time }\end{array}$ & Priority & $\begin{array}{l}\text { Waiting } \\
\text { Time }\end{array}$ & $\begin{array}{l}\text { Turnaround } \\
\text { time }\end{array}$ \\
\hline P1 & 7 & 2 & 5 & 12 \\
\hline P2 & 5 & 1 & o & 5 \\
\hline P3 & 3 & 4 & 20 & 23 \\
\hline P4 & 8 & 3 & 12 & 20 \\
\hline P2 & P1 & \multicolumn{2}{|l|}{ P4 } & P3 \\
\hline 0 & \multicolumn{3}{|c|}{12} & 20 \\
\hline
\end{tabular}

Figure 4 Priority Scheduling

waiting time for process $\mathrm{P} 1=5, \mathrm{P} 2=0, \mathrm{P} 3=20, \mathrm{P} 4=12$.

Average waiting time $=(5+0+20+12) / 4=9.25$

Average TurnaroundTime $=(12+5+23+20) / 4=15$

\section{ROUND ROBIN SCHEDULING}

The processes are scheduled using FCFS algorithm but each process is given with time quantum or time slice. Each process will execute for that amount of time slice and switches to next process in the FCFS order till their completion.[8] The table 4 lists the processes and their execution time (burst time) and time slice is given as 3 time units. The round robin scheduling (Figure 5) is applied and the average waiting time and turnaround time are calculated and it is listed in table 4.

Table 4 Process and their burst times

\begin{tabular}{|c|c|c|c|c|c|c|c|c|}
\hline \multicolumn{2}{|c|}{ Process } & \multicolumn{2}{|c|}{ Burst Time } & \multicolumn{2}{|c|}{ Waiting Time } & \multicolumn{3}{|c|}{ Turnaround time } \\
\hline \multicolumn{2}{|c|}{$\mathbf{P 1}$} & & 7 & & 14 & \multicolumn{3}{|c|}{21} \\
\hline \multicolumn{2}{|c|}{ P2 } & & 5 & & 12 & \multicolumn{3}{|c|}{17} \\
\hline \multicolumn{2}{|c|}{ P3 } & & 3 & & 6 & \multicolumn{3}{|c|}{9} \\
\hline \multicolumn{2}{|c|}{ P4 } & \multicolumn{2}{|c|}{8} & & 15 & \multicolumn{3}{|c|}{23} \\
\hline P1 & P2 & P3 & P4 & P1 & P2 & 4 & P1 & P4 \\
\hline
\end{tabular}


$\begin{array}{llllllllll}0 & 3 & 6 & 9 & 12 & 15 & 17 & 20 & 21 & 23\end{array}$

Figure 5 RoundRobin Scheduling

waiting time for process $\mathrm{P} 1=14, \mathrm{P} 2=12, \mathrm{P} 3=6, \mathrm{P} 4=15$.

Average WaitingTime $=(14+12+6+15) / 4=11.75$

Average Turnaround Time $=(21+17+9+23) / 4=17.5$

\section{DESIGN OF OPTIMAL SCHEDULER}

The job of optimal scheduler is to schedule the jobs in such a way that it gives minimum average waiting time and turnaround time. The proposed scheduler (Figure 6) selects the Round Robin algorithm if the time slice input is available. Otherwise it checks for the priority input. If available it goes for priority algorithm. If both are not available the SJF algorithm is selected by the scheduler because SJF gives minimum waiting time and turnaround time when compare to FCFS.

\section{RESULT AND DISCUSSION}

The various scheduling algorithms are applied to the same problem and the results are compared. From table 5 and Figure 7 it is clear that SJF algorithm gives better performance compared to other algorithms.

Table 5 Average waiting time and turnaround time using various algorithms

\begin{tabular}{|l|c|c|}
\hline Algorithm & $\begin{array}{l}\text { Average Waiting } \\
\text { Time (time units) }\end{array}$ & $\begin{array}{l}\text { Average Turnaround time } \\
\text { (time units) }\end{array}$ \\
\hline FCFS & 8.5 & 14.25 \\
\hline SJF & 6.5 & 12.25 \\
\hline Priority & 9.25 & 15 \\
\hline Round Robin & 11.75 & 17.5 \\
\hline
\end{tabular}

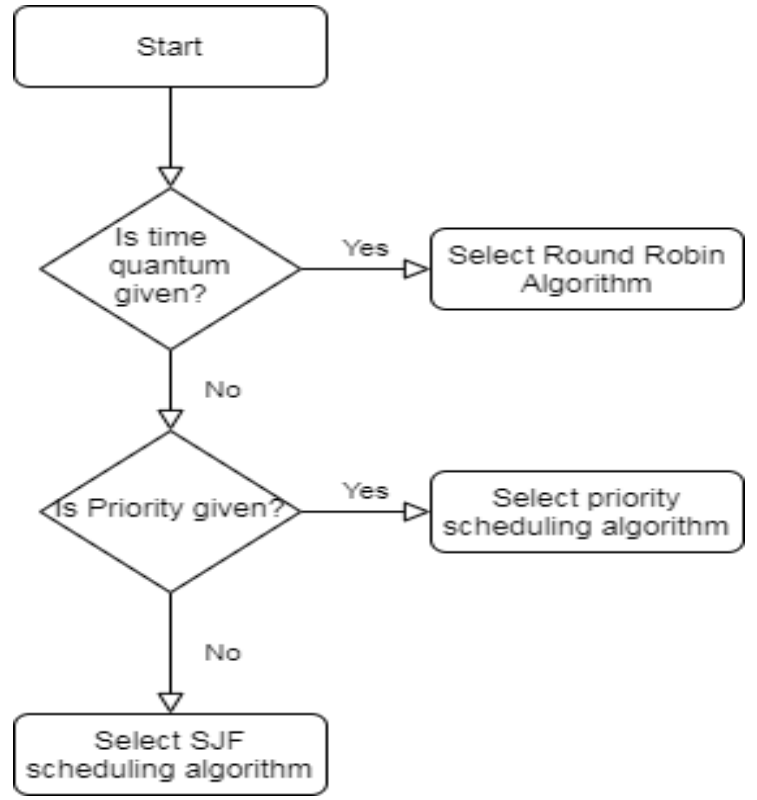

Figure 6 Optimal Scheduler

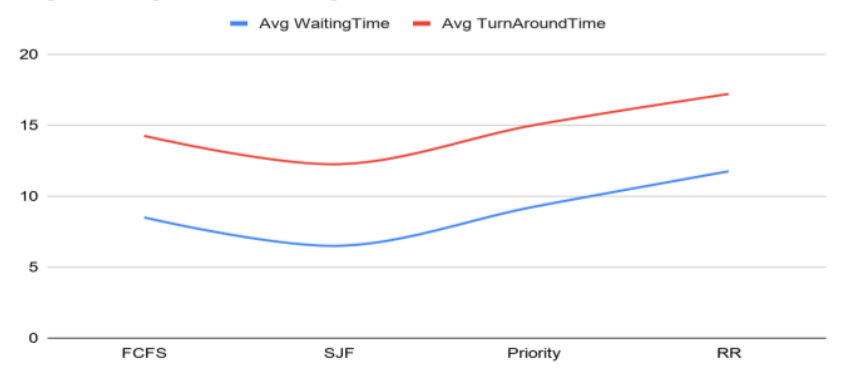

Figure 7 Average waiting time and turnaround time for different algorithms

\section{CONCLUSION}

The Optimal CPU scheduler is designed based on the results of applying the different scheduling algorithms to the same problem. By default SJF algorithm is selected as the best algorithm by the scheduler. If the time quantum is given then the scheduler selects RoundRobin algorithm for scheduling. If the priority is given then the priority algorithm will be selected for scheduling.

\section{REFERENCES}

1. Choudhary, N., Gautam, G., Goswami, Y., \& Khandelwal, S. (2019). A Comparative Study of Various CPU Scheduling Algorithms using MOOS Simulator. Journal of Advances in Shell Programming, 5(3), $1-5$.

2. Jain, S., \& Jain, S. (2016). A review study on the CPU scheduling algorithms. International Journal of Advanced Research in Computer and Communication Engineering, 5(8), 22-31.

3. Chandra Shekar, N., and V. Karthik. "Analysis of Priority Scheduling Algorithm on the Basis of FCFS \& SJF for Similar Priority Jobs." International Journal of Engineering Research in Computer Science and Engineering (IJERCSE) 4.3 (2017).

4. Aslam, Naila. Designing a Model for improving CPU Scheduling by using Machine Learning. Diss. 2017.

5. Sowmya, G., et al. "A Comparison Of Scheduling Algorithm For Best Utilization Of Memory." International Journal of Pure and Applied Mathematics 120.6 (2018): 3563-3570.

6. Sankar, A., and G. Ananthnath. "Improved Dynamic Time Slice Priority Scheduling Algorithm with Unknown Burst Time." (2018).

7. Joon, Monika, and Neetu Sharma. "Optimization Of Scheduling Algorithms For Load Balancing Using Hybrid Approach." (2017).

8. Abraham Silberschatz, Peter Baer Galvin and Greg Gagne, "Operating System Concepts", Ninth Edition, 2013.

\section{AUTHORS PROFILE}

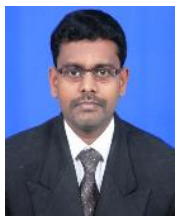

Mr. Sambath M. is graduated in Computer Science \& Engineering in the year 2001 and obtained his Master degree in Computer Science \& Engineering from Anna University in the year 2008 and currently pursuing his Ph.D. at Hindustan Institute of Technology and Science, Chennai, INDIA. His area of interest includes Image processing, Algorithms, Operating systems.

Dr. D. John Aravindhar received Ph.D. degree in Data mining from Hindustan Institute of Technology and Science. He is currently a Professor in Computer Science Department of Hindustan Institute of Technology and Science.His area of interest are Data mining and cloud computing.

P. Harshavardhan Reddy, Lokesh kumar Ravipati, Suthapalli Sai gowtham, Korrapati Jagadeesh, Vemula Raviteja are currently pursuing their B.Tech in Computer Science and Engineering at Hindustan Institute of Technology and Science, Chennai,

INDIA.

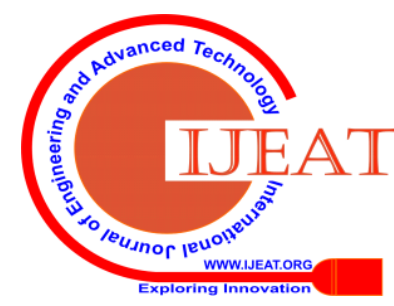

